\title{
LONGITUDINAL ELECTRON BUNCH DIAGNOSTICS USING COHERENT TRANSITION RADIATION
}

\author{
D. Mihalcea, Northern Illinois University, DeKalb, IL 60115, USA \\ C. L. Bohn, Northern Illinois University, DeKalb, IL 60115, USA \\ U. Happek, University of Georgia, Athens, GA 30602, USA \\ P. Piot, Fermilab, Batavia, IL 60510-0500, USA
}

\begin{abstract}
The longitudinal charge distribution of electron bunches in the Fermilab/Nicadd A0 photoinjector was determined using the coherent transition radiation produced by electrons passing through a thin metallic foil. The autocorrelation of the transition radiation signal was measured with a Michelson-type interferometer. The response function of the interferometer was determined from measured and simulated power spectra for low electron bunch charge and maximum longitudinal compression. A Kramers-Kronig technique was used to determine longitudinal charge distribution. Measurements were performed for electron bunch lengths in the range from 0.3 to $2 \mathrm{ps}$ (rms).
\end{abstract}

\section{INTRODUCTION}

Drive linacs for free electron lasers, functioning in the vacuum ultra-violet or X-ray range, require sub-picosecond accelerated electron bunches [1]. In many cases such short bunches are obtained by compressing the beam in magnetic chicanes and, as a result, the longitudinal charge distribution is significantly distorted from its original gaussian shape. Therefore, in order to determine parameters like peak current or FWHM, it is important to have a complete measurement of the bunch longitudinal charge distribution rather than just an estimate of its length.

Coherent transition radiation (CTR) emitted by electron bunches when crossing a metallic foil can be related to the longitudinal charge distribution form factor $f(\omega)[2,3]$ :

$$
I(\omega)=N(N-1) I_{e}|f(\omega)|^{2}
$$

where $\mathrm{N}$ is the total number of electrons in the bunch and $I_{e}$ is the radiation emitted by a single electron. In the limit of perfect conducting metallic foil, $I_{e}$ does not depend on frequency. The form factor $f(\omega)$ is related to the normalized longitudinal charge distribution $\rho(z)$ :

$$
f(\omega)=\int \rho(z) \exp (i \omega z / c) d z
$$

Since only the power spectrum $I(\omega)$ can be experimentally measured, the phase of the form factor is unknown. However, the frequency-dependent phase of the form factor can be well approximated if $I(\omega)$ is known for the entire wavelength spectrum (Kramers-Kroning method [4]):

$$
\psi(\omega)=-\frac{2 \omega}{\pi} \int_{0}^{\infty} d x \frac{\ln [\kappa(x) / \kappa(\omega)]}{x^{2}-\omega^{2}}
$$

where $\kappa(\omega) \equiv \sqrt{I(\omega)}$. With this approximation the normalized longitudinal charge distribution is given by:

$$
\rho(z)=\frac{1}{\pi c} \int_{0}^{\infty} \kappa(\omega) \cos [\psi(\omega)-\omega z / c] d \omega
$$

Longitudinal profile measurements were performed at FNPL (Fermilab/NICADD Photoinjector Laboratory). Typically, the electron energy is about $16 \mathrm{MeV}$ and the bunch rms length ranges from 3 to 10 ps when bunch charge is a few nanocoulombs. Electron bunches are compressed in the sub-picosecond range with a four-dipole magnetic chicane located downstream from the accelerating structures.

\section{MICHELSON INTERFEROMETER}

CTR is produced by electron bunches crossing an aluminium foil oriented at $45^{\circ}$ with respect to the beamline. Between the aluminium foil and the Michelson interferometer, shown schematically in Fig. 1, there are a singlecrystal quartz window and two concave gold-coated mirrors which convert the divergent beam to a parallel one.

The beam splitter is coated with a thin inconel layer of thickness chosen such that transmission and reflection are $30 \%$ and independent of the wavelength up to the plasma frequency. One arm of the interferometer has a mirror mounted on a translation stage which motion can be remotely controlled. The right arm of the interferometer contains a semi-transparent mirror which has constant $30 \%$ transmission and $30 \%$ reflection coefficients within the relevant wavelength range. Transmitted radiation is focused on the reference detector by a concave mirror. The combined radiation from the movable mirror and from the reflection on the semi-transparent mirror is focused on the detector in the left arm of the interferometer.

The autocorrelation signal is the superposition of the time-dependent electric fields from the two arms of the interferometer, and it is recorded by the first detector as a function of the path difference $\delta$ :

$$
I_{1} \propto \int_{-\infty}^{+\infty}|E(t+\delta / c)+E(t)|^{2} d t
$$




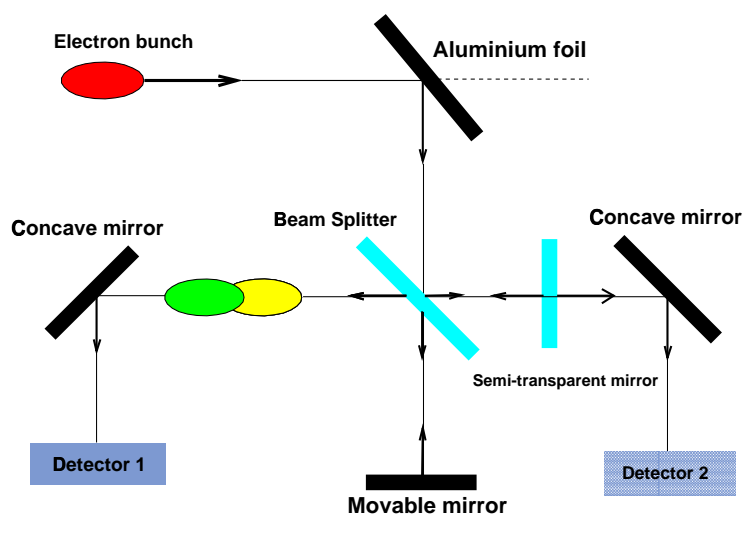

Figure 1: Michelson interferometer. Detector 1 is used to record the autocorrelation function. Detector 2 is a reference detector used to normalize the autocorrelation function.

The intensity recorded by the second detector does not depend on path difference but it is sensitive to beam instabilities as well as the first detector. Therefore it is convenient to define the autocorrelation function $S(\delta)$ as the ratio of the intensities measured by the two detectors:

$$
S(\delta) \equiv \frac{I_{1}}{I_{2}} \propto \frac{\operatorname{Re} \int E(t) E^{*}(t+\delta / c) d t}{\int|E(t)|^{2} d t}
$$

In our latest measurements the intesity $I_{1}$ was measured with a Golay cell and the reference intensity $I_{2}$ with a less expensive Molectron pyroelectric detector.

\section{RESULTS}

During a data-taking session the position of the movable mirror is changed with a predefined step (typically $50 \mu \mathrm{m}$ ). At each position of the movable mirror, the intensities $I_{1}$ and $I_{2}$ (Eq. 6) are measured. Typically, 5 such measurements are performed and average values of $I_{1}$ and $I_{2}$, as well as their $\sigma$ 's are recorded. The intensity of the UV laser varies from shot to shot and so does the electron bunch charge. These variations are up to $50 \%$ of the nominal laser intensity. Since the intensity of the coherent radiation depends on the electron bunch charge (Eq. 1), the intensities $I_{1}$ and $I_{2}$ are measured only if the bunch charge is within some narrow limits, typically less than $10 \%$ away from the nominal charge. The autocorrelation and power spectrum (Fourier transform of the autocorrelation) are shown in Fig. 2 for $1 \mathrm{nC}$ electron bunches at maximum compression.

The measured power spectra differ from what theoretical considerations predict (see Eqn.1). The reason is that measured power spectra incorporate a filter function specific to the measuring devices. At lower frequencies $(<0.1 \mathrm{THz})$ measured power spectra are depleted due to detectors' low
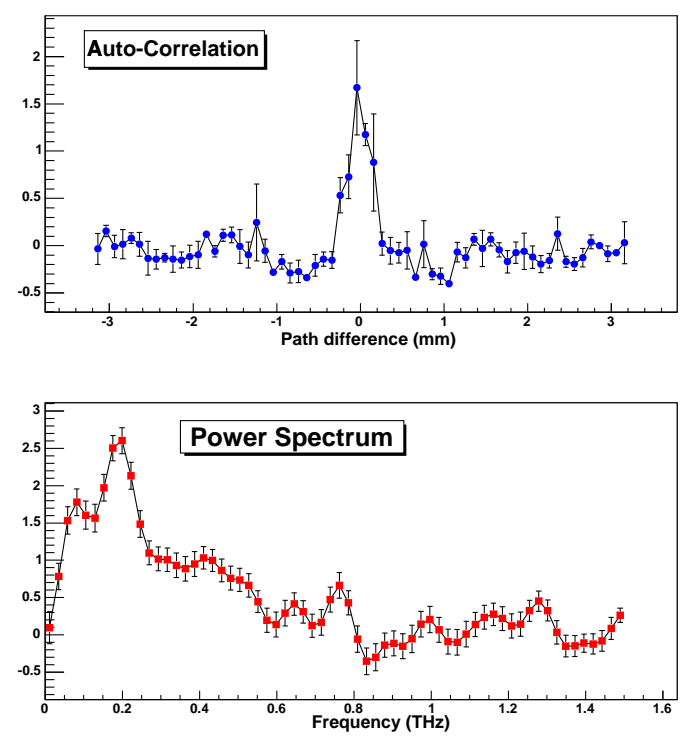

Figure 2: Autocorrelation function and power spectrum for $1 \mathrm{nC}$ electron bunches at maximum compression.

sensitivity in this spectral range, and to diffraction on optical components. At higher frequencies $(>0.5 \mathrm{THz})$, part of the CTR is absorbed by the quartz window and other optical components. A direct measurement of the interferometer's response function would be ideal but it is experimentally very challenging and expensive (Ref. [5]).

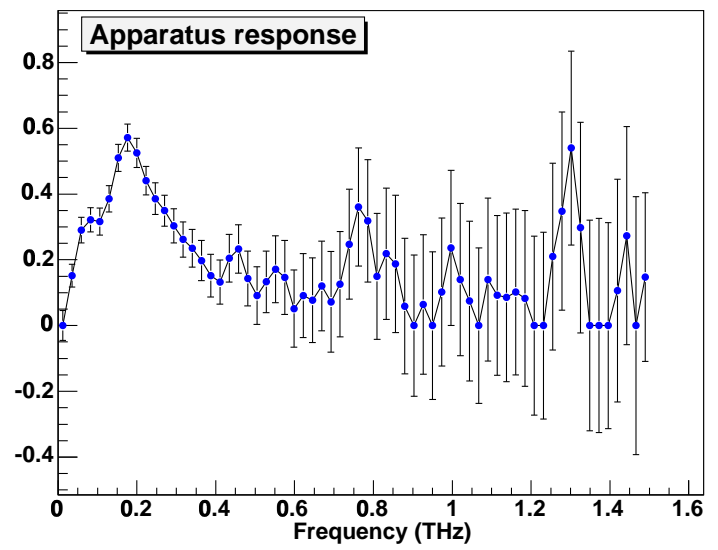

Figure 3: Interferometer response function obtained as ratio of experimental power spectrum and simulated power spectrum for $1 \mathrm{nC}$ electron bunches at maximum compression.

A much simpler alternative is to choose some well defined and easy to reproduce experimental conditions, and to assume that in these particular circumstances a tracking code (in our case Parmela) correctly simulates the longitudinal electron bunch shape. In "standard" experimental conditions, electron bunches of $1 \mathrm{nC}$ are generated by 
laser pulses of gaussian shape ( $\sigma \approx 3.5 \mathrm{ps}$ ), accelerated to $\approx 14 \mathrm{MeV}$, and maximally compressed by the magnetic chicane. The response function is obtained by dividing the experimental power spectrum to the simulated one (Fig. 3).

The interferometer's response function is used to correct power spectra when electron bunches are produced under some arbitrary experimental conditions. Corrected power spectra still do not cover the whole spectral range. The reason is that interferometer's response function is close to zero at low and high frequencies. Therefore, in order to apply the Kramers-Kroning method (Eq. 4), the corrected power spectrum must be completed for the missing frequencies. Power spectra are completed, for low and high frequencies, by using the asymptotic expressions derived in Ref. [4]. The power spectrum completion procedure is plagued by uncertainties, because it is not obvious what "low and high frequencies" really means, and interpolation procedures are somewhat arbitrary. Fig. 4 shows the autocorrelation and completed power spectrum for $3.2 \mathrm{nC}$ electron bunches at a moderate compression.
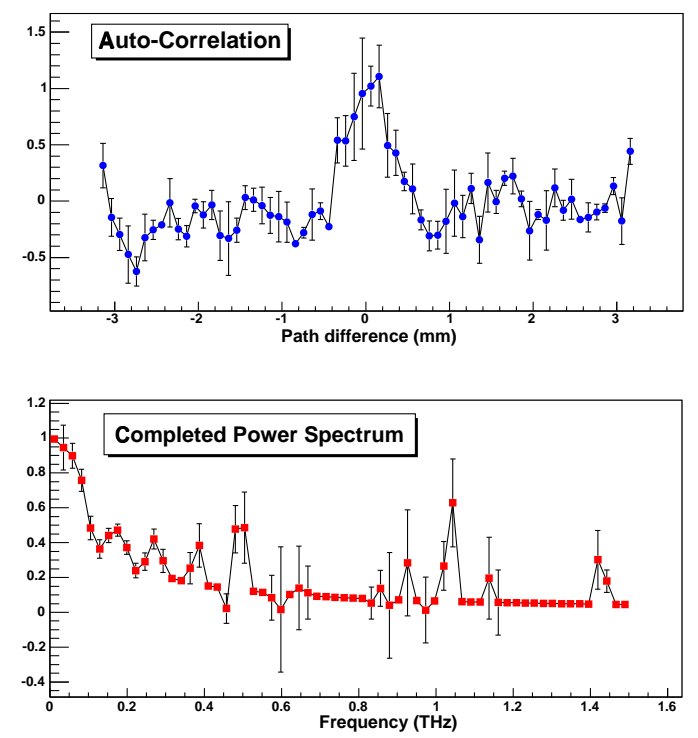

Figure 4: Autocorrelation (top) and completed power spectrum (bottom) for $3.2 \mathrm{nC}$ electron bunches at moderate compression.

Larger electron bunch charges were obtained by increasing the laser intensity and keeping constant its duration. The length of electron bunches is higher in the case shown in Fig. 4 compared with the case shown in Fig. 2, because the bunch charge is larger and also because the level of compression is lower. Larger bunch length translates into wider autocorrelation function and narrower power spectrum.

The longitudinal electron bunch shape is reconstructed by using the Kramers-Kroning method (Eq. 3). An example of such reconstruction is shown in Fig. 5 where the Kramers-Kroning technique was applied to the completed

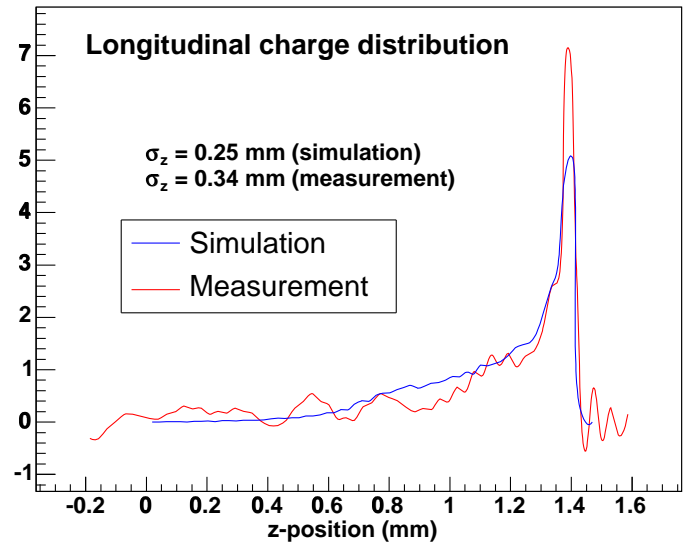

Figure 5: Longitudinal charge distribution for $3.2 \mathrm{nC}$ electron bunches at moderate compression reconstructed from experimental data (red line) and from Parmela simulation (blue line). The high charge distribution peak is at the head of the electron bunch.

power spectrum shown in Fig. 4. The agreement between measurement and Parmela simulation is within 30\% for bunch length and peak height. High charge density at the head of the electron bunch is a direct effect of the compression. The width of the peak, as well as the shape and the length of the charge distribution tail can be related to energy spread, nonlinearity of the rf field, and to bunch compressor parameters $R_{56}$ and $T_{566}$ (Ref. [6]).

\section{CONCLUSIONS}

So far we measured longitudinal charge density profiles for electron bunches that have a simple gaussian shape before compression. Agreement with simulation is quite good. There are two major sources of systematic uncertainties in this analysis: (a) the inaccuracy of the interferometer's response function, and (b) the ambiguities of the power spectrum completion procedure. We plan to use electron bunches with some longitudinal spatial structure to estimate how much systematic uncertainties affect the results. For example, a good way to prove the reliability of this method is to reconstruct bunches consisting of two pulses separated by a known distance (Ref. [7]).

\section{REFERENCES}

[1] V. Avyazian, et al. Eur. Phys. J. D. 20, 149-155 (2002).

[2] J. S. Nodvick, D. S. Saxon, Physical Review, 96, 180 (1954).

[3] C. J. Hirschmugl, et al. Physical Review A, 44, 1316 (1991).

[4] R. Lai, A. J. Sievers, Physical Review, E50, R3342, (1994).

[5] O. G. Grimm, et al. Proceedings of the 2004 FEL Conference, p 395-398.

[6] R. Li, NIM A475, 498 (2001).

[7] R. Tikhoplav, et al. Proceedings of LINAC 2004, p 149. 